\title{
Some New Saigo Type Fractional Integral Inequalities and Their q-Analogues
}

\author{
Junesang Choi ${ }^{1}$ and Praveen Agarwal ${ }^{2}$ \\ ${ }^{1}$ Department of Mathematics, Dongguk University, Gyeongju 780-714, Republic of Korea \\ ${ }^{2}$ Department of Mathematics, Anand International College of Engineering, Jaipur-303012, India
}

Correspondence should be addressed to Junesang Choi; junesang@mail.dongguk.ac.kr

Received 6 January 2014; Accepted 21 February 2014; Published 2 April 2014

Academic Editor: G. Wang

Copyright (C) 2014 J. Choi and P. Agarwal. This is an open access article distributed under the Creative Commons Attribution License, which permits unrestricted use, distribution, and reproduction in any medium, provided the original work is properly cited.

The main objective of this paper is to establish some, presumably, new Saigo type fractional integral inequalities whose special cases are shown to yield corresponding inequalities associated with Riemann-Liouville and Erdélyi-Kober type fractional integral operators, respectively. We also investigate and present $q$-extensions of the above results and some other presumably new ones. Relevant connections of the results presented here with those earlier ones are also indicated.

\section{Introduction and Preliminaries}

Throughout this paper, $\mathbb{N}, \mathbb{R}, \mathbb{C}$, and $\mathbb{Z}_{0}^{-}$denote the sets of positive integers, real numbers, complex numbers, and nonpositive integers, respectively, and $\mathbb{N}_{0}:=\mathbb{N} \cup\{0\}$. Consider the following functional:

$$
\begin{aligned}
T(f, g, p, q)= & \int_{a}^{b} q(x) d x \int_{a}^{b} p(x) f(x) g(x) d x \\
& +\int_{a}^{b} p(x) d x \int_{a}^{b} q(x) f(x) g(x) d x \\
& -\left(\int_{a}^{b} q(x) f(x) d x\right)\left(\int_{a}^{b} p(x) g(x) d x\right) \\
& -\left(\int_{a}^{b} p(x) f(x) d x\right)\left(\int_{a}^{b} q(x) g(x) d x\right),
\end{aligned}
$$

where $f, g:[a, b] \rightarrow \mathbb{R}$ are two integrable functions on $[a, b]$ and $p(x)$ and $q(x)$ are positive integrable functions on $[a, b]$. If $f$ and $g$ are synchronous on $[a, b]$, that is,

$$
(f(x)-f(y))(g(x)-g(y)) \geq 0,
$$

for any $x, y \in[a, b]$, then we have (see, e.g., $[1,2]$ )

$$
T(f, g, p, q) \geq 0 .
$$

The inequality in (2) is reversed, if $f$ and $g$ are asynchronous on $[a, b]$, that is,

$$
(f(x)-f(y))(g(x)-g(y)) \leq 0,
$$

for any $x, y \in[a, b]$. If $p(x)=q(x)$, for any $x, y \in[a, b]$, we get the Chebyshev inequality (see [3]). Ostrowski [4] established the following generalization of the Chebyshev inequality.

If $f$ and $g$ are two differentiable and synchronous functions on $[a, b]$ and $p$ is a positive integrable function on $[a, b]$ with $\left|f^{\prime}(x)\right| \geq m \geq 0$ and $\left|g^{\prime}(x)\right| \geq r \geq 0$, for $x \in[a, b]$, then we have

$$
T(f, g, p)=T(f, g, p, p) \geq m r T(x-a, x-a, p) \geq 0 .
$$

If $f$ and $g$ are asynchronous on $[a, b]$, then we have

$$
T(f, g, p) \leq \operatorname{mr} T(x-a, x-a, p) \leq 0 .
$$

If $f$ and $g$ are two differentiable functions on $[a, b]$ with $\left|f^{\prime}(x)\right| \leq M$ and $\left|g^{\prime}(x)\right| \leq R$, for $x \in[a, b]$, and $p$ is a positive integrable function on $[a, b]$, then we have

$$
|T(f, g, p)| \leq \operatorname{MRT}(x-a, x-a, p) \leq 0 .
$$


The functional (1) has attracted many researchers' attention due mainly to diverse applications in numerical quadrature, transform theory, and probability and statistical problems. Among those applications, the functional (1) has also been employed to yield a number of integral inequalities (see, e.g., [5-12]; for a very recent work, see also [13]).

Here, in this paper, we aim at establishing certain, presumably, new inequalities involving Saigo fractional integral operator (8) whose special cases are shown to yield corresponding inequalities associated with Riemann-Liouville fractional integral operator (11) and Erdélyi-Kober fractional integral operator (12). We also investigate and present $q$ extensions of our results and some other presumably new ones. Relevant connections of some of the results presented here with those earlier ones are also pointed out.

For our purpose, we also need to recall the following definitions and some earlier works.

Definition 1. A real-valued function $f(t)(t>0)$ is said to be in the space $C_{\mu}(\mu \in \mathbb{R})$, if there exists a real number $p>\mu$ such that $f(t)=t^{p} \phi(t)$, where $\phi(t) \in C(0, \infty)$.

Definition 2. A function $f(t)(t>0)$ is said to be in the space $C_{\mu}^{n}(n \in \mathbb{R})$, if $f^{(n)} \in C_{\mu}$.

Definition 3. Let $\alpha>0$ and $\beta, \eta \in \mathbb{R}$. Then the Saigo fractional integral $I_{0, t}^{\alpha, \beta, \eta}$ (in terms of the Gauss hypergeometric function) of order $\alpha$ for a real-valued continuous function $f(t)$ is defined by (see [14]; see also [15])

$$
\begin{aligned}
& I_{0, t}^{\alpha, \beta, \eta}\{f(t)\}=\frac{t^{-\alpha-\beta}}{\Gamma(\alpha)} \int_{0}^{t}(t-\tau)^{\alpha-1}{ }_{2} F_{1} \\
& \times\left(\alpha+\beta,-\eta ; \alpha ; 1-\frac{\tau}{t}\right) f(\tau) d \tau,
\end{aligned}
$$

where the function ${ }_{2} F_{1}(\cdot)$ is the Gaussian hypergeometric function defined by (see, e.g., [16, Section 1.5])

$$
{ }_{2} F_{1}(a, b ; c ; t)=\sum_{n=0}^{\infty} \frac{(a)_{n}(b)_{n}}{(c)_{n}} \frac{t^{n}}{n !}
$$

and $\Gamma(\alpha)$ is the familiar Gamma function. Here, $(a)_{n}$ is the Pochhammer symbol defined, for $a \in \mathbb{C}$, by (see, e.g., [16, p. 2 and pp. 4-6])

$$
\begin{aligned}
(a)_{n} & := \begin{cases}1 & (n=0) \\
a(a+1) \cdots(a+n-1) & (n \in \mathbb{N})\end{cases} \\
& =\frac{\Gamma(a+n)}{\Gamma(a)} \quad\left(a \in \mathbb{C} \backslash \mathbb{Z}_{0}^{-}\right) .
\end{aligned}
$$

It is noted that Saigo fractional integral operator $I_{0, t}^{\alpha, \beta, \eta}$ includes both Riemann-Liouville and Erdélyi-Kober fractional integral operators, respectively, given by the following relationships (see, e.g., [17]), for $f \in C_{\mu}(\mu \geq-1)$ :

$$
\begin{aligned}
& I_{0, t}^{\alpha}\{f(t)\}:(\left.=I_{0, t}^{\alpha,-\alpha, \eta}\{f(t)\}\right) \\
&=\frac{1}{\Gamma(\alpha)} \int_{0}^{t}(t-\tau)^{\alpha-1} f(\tau) d \tau \quad(\alpha>0), \\
& I_{0, t}^{\alpha, \eta}\{f(t)\}:(\left.=I_{0, t}^{\alpha, 0, \eta}\{f(t)\}\right) \\
&=\frac{t^{-\alpha-\eta}}{\Gamma(\alpha)} \int_{0}^{t}(t-\tau)^{\alpha-1} \tau^{\eta} f(\tau) d \tau \\
& \quad(\alpha>0 ; \eta \in \mathbb{R}) .
\end{aligned}
$$

For our purpose, we also recall the following definitions (see, e.g., [16, Section 6]) and some earlier works.

The $q$-shifted factorial $(a ; q)_{n}$ is defined by

$$
(a ; q)_{n}:= \begin{cases}1 & (n=0) \\ \prod_{k=0}^{n-1}\left(1-a q^{k}\right) & (n \in \mathbb{N}),\end{cases}
$$

where $a, q \in \mathbb{C}$ and it is assumed that $a \neq q^{-m}\left(m \in \mathbb{N}_{0}\right)$.

The $q$-shifted factorial for negative subscript is defined by

$$
\begin{array}{r}
(a ; q)_{-n}:=\frac{1}{\left(1-a q^{-1}\right)\left(1-a q^{-2}\right) \cdots\left(1-a q^{-n}\right)} \\
\left(n \in \mathbb{N}_{0}\right) .
\end{array}
$$

We also write

$$
(a ; q)_{\infty}:=\prod_{k=0}^{\infty}\left(1-a q^{k}\right) \quad(a, q \in \mathbb{C} ;|q|<1) .
$$

It follows from (13), (14), and (15) that

$$
(a ; q)_{n}=\frac{(a ; q)_{\infty}}{\left(a q^{n} ; q\right)_{\infty}} \quad(n \in \mathbb{Z}),
$$

which can be extended to $n=\alpha \in \mathbb{C}$ as follows:

$$
(a ; q)_{\alpha}=\frac{(a ; q)_{\infty}}{\left(a q^{\alpha} ; q\right)_{\infty}} \quad(\alpha \in \mathbb{C} ;|q|<1),
$$

where the principal value of $q^{\alpha}$ is taken.

We begin by noting that F. J. Jackson was the first to develop $q$-calculus in a systematic way. The $q$-derivative of a function $f(t)$ is defined by

$$
D_{q}\{f(t)\}:=\frac{d_{q}}{d_{q} t}\{f(t)\}=\frac{f(q t)-f(t)}{(q-1) t} .
$$

It is noted that

$$
\lim _{q \rightarrow 1} D_{q}\{f(t)\}=\frac{d}{d t}\{f(t)\},
$$

if $f(t)$ is differentiable. 
The function $F(t)$ is a q-antiderivative of $f(t)$, if $D_{q}\{F(t)\}=f(t)$. It is denoted by

$$
\int f(t) d_{q} t
$$

The Jackson integral of $f(t)$ is thus defined, formally, by

$$
\int f(t) d_{q} t:=(1-q) t \sum_{j=0}^{\infty} q^{j} f\left(q^{j} t\right)
$$

which can be easily generalized as follows:

$$
\int f(t) d_{q} g(t)=\sum_{j=0}^{\infty} f\left(q^{j} t\right)\left(g\left(q^{j} t\right)-g\left(q^{j+1} t\right)\right) .
$$

Suppose that $0<a<b$. The definite $q$-integral is defined as follows:

$$
\begin{gathered}
\int_{0}^{b} f(t) d_{q} t:=(1-q) b \sum_{j=0}^{\infty} q^{j} f\left(q^{j} b\right), \\
\int_{a}^{b} f(t) d_{q} t=\int_{0}^{b} f(t) d_{q} t-\int_{0}^{a} f(t) d_{q} t .
\end{gathered}
$$

A more general version of (23) is given by

$$
\int_{0}^{b} f(t) d_{q} g(t)=\sum_{j=0}^{\infty} f\left(q^{j} b\right)\left(g\left(q^{j} b\right)-g\left(q^{j+1} b\right)\right) .
$$

The classical Gamma function $\Gamma(z)$ (see, e.g., [16, Section 1.1]) was found by Euler, while he was trying to extend the factorial $n !=\Gamma(n+1)\left(n \in \mathbb{N}_{0}\right)$ to real numbers. The $q$ factorial function $[n]_{q} !\left(n \in \mathbb{N}_{0}\right)$ of $n$ !, defined by

$$
[n]_{q} !:= \begin{cases}1 & \text { if } n=0, \\ {[n]_{q}[n-1]_{q} \cdots[2]_{q}[1]_{q}} & \text { if } n \in \mathbb{N},\end{cases}
$$

can be rewritten as follows:

$$
\begin{aligned}
(1-q) \prod_{k=0}^{-n} \frac{\left(1-q^{k+1}\right)}{\left(1-q^{k+1+n}\right)} & =\frac{(q ; q)_{\infty}}{\left(q^{n+1} ; q\right)_{\infty}}(1-q)^{-n} \\
& :=\Gamma_{q}(n+1) \quad(0<q<1) .
\end{aligned}
$$

By replacing $n$ by $a-1$ in (27), Jackson [18] defined the $q$ Gamma function $\Gamma_{q}(a)$ by

$$
\Gamma_{q}(a):=\frac{(q ; q)_{\infty}}{\left(q^{a} ; q\right)_{\infty}}(1-q)^{1-a} \quad(0<q<1) .
$$

The $q$-analogue of $(t-a)^{n}$ is defined by the polynomial

$$
\begin{aligned}
(t-a)_{q}^{n} & := \begin{cases}1 & (n=0) \\
(t-a)(t-q a) \cdots\left(t-q^{n-1} a\right) & (n \in \mathbb{N})\end{cases} \\
& =t^{n}\left(\frac{a}{t} ; q\right)_{n} \quad\left(n \in \mathbb{N}_{0}\right) .
\end{aligned}
$$

Definition 4. Let $\mathfrak{R}(\alpha)>0$; let $\beta$ and $\eta$ be real or complex numbers. Then a $q$-analogue of Saigo's fractional integral $I_{q}^{\alpha, \beta, \eta}$ is given for $|\tau / t|<1$ by (see [19, p. 172, Equation (2.1)])

$$
\begin{aligned}
I_{q}^{\alpha, \beta, \eta}\{f(t)\}:= & \frac{t^{-\beta-1}}{\Gamma_{q}(\alpha)} \\
& \times \int_{0}^{t}\left(\frac{q \tau}{t} ; q\right)_{\alpha-1} \sum_{m=0}^{\infty} \frac{\left(q^{\alpha+\beta} ; q\right)_{m}\left(q^{-\eta} ; q\right)_{m}}{\left(q^{\alpha} ; q\right)_{m}(q ; q)_{m}} \\
& \cdot q^{(\eta-\beta) m}(-1)^{m} q^{-\left(\begin{array}{c}
m \\
2
\end{array}\right)\left(\frac{\tau}{t}-1\right)_{q}^{m} f(\tau) d_{q} \tau .}
\end{aligned}
$$

The integral operator $I_{q}^{\alpha, \beta, \eta}$ includes both the $q$-analogues of the Riemann-Liouville and Erdélyi-Kober fractional integral operators given by the following definitions.

Definition 5. A q-analogue of Riemann-Liouville fractional integral operator of a function $f(t)$ of an order $\alpha$ is given by (see [20])

$$
\begin{aligned}
I_{q}^{\alpha}\{f(t)\}:( & \left.=I_{q}^{\alpha,-\alpha, \eta}\{f(t)\}\right) \\
= & \frac{t^{\alpha-1}}{\Gamma_{q}(\alpha)} \int_{0}^{t}\left(\frac{q \tau}{t} ; q\right)_{\alpha-1} f(\tau) d_{q} \tau \\
& (\alpha>0 ; 0<q<1),
\end{aligned}
$$

where $(a ; q)_{\alpha}$ is given by (17).

Definition 6. A q-analogue of the Erdélyi-Kober fractional integral operator for $\alpha>0, \eta \in \mathbb{R}$, and $0<q<1$ is given by (see [20])

$$
\begin{aligned}
I_{q}^{\eta, \alpha}\{f(t)\}:( & \left.=I_{q}^{\alpha, 0, \eta}\{f(t)\}\right) \\
& =\frac{t^{-\eta-1}}{\Gamma_{q}(\alpha)} \int_{0}^{t}\left(\frac{q \tau}{t} ; q\right)_{\alpha-1} \tau^{\eta} f(\tau) d_{q} \tau
\end{aligned}
$$

$$
(\alpha>0 ; 0<q<1) .
$$

\section{Certain Inequalities Involving Saigo Fractional Integral Operator}

Here, we start with presenting an inequality involving Saigo fractional integral (8) asserted by the following lemma.

Lemma 7. Let $f$ and $g$ be two continuous and synchronous functions on $[0, \infty)$ and let $u, v:[0, \infty) \rightarrow[0, \infty)$ be continuous functions. Then the following inequality holds true:

$$
\begin{aligned}
I_{0, t}^{\alpha, \beta, \eta}\{u(t)\} I_{0, t}^{\alpha, \beta, \eta}\{v(t) f(t) g(t)\} \\
+I_{0, t}^{\alpha, \beta, \eta}\{v(t)\} I_{0, t}^{\alpha, \beta, \eta}\{u(t) f(t) g(t)\} \\
\geq I_{0, t}^{\alpha, \beta, \eta}\{u(t) f(t)\} I_{0, t}^{\alpha, \beta, \eta}\{v(t) g(t)\} \\
\quad+I_{0, t}^{\alpha, \beta, \eta}\{v(t) f(t)\} I_{0, t}^{\alpha, \beta, \eta}\{u(t) g(t)\},
\end{aligned}
$$

for all $t>0, \alpha>0$, and $\beta, \eta \in \mathbb{R}$ with $\alpha+\beta \geqq 0$ and $\eta \leqq 0$. 
Proof. Let $f$ and $g$ be two continuous and synchronous functions on $[0, \infty)$. Then, for all $\tau, \rho \in(0, t)$ with $t>0$, we have

$$
(f(\tau)-f(\rho))(g(\tau)-g(\rho)) \geq 0
$$

or, equivalently,

$$
f(\tau) g(\tau)+f(\rho) g(\rho) \geq f(\tau) g(\rho)+f(\rho) g(\tau) .
$$

Consider the following function $F(t, \tau)$ defined by

$$
\begin{aligned}
& F(t, \tau)= \frac{t^{-\alpha-\beta}(t-\tau)^{\alpha-1}}{\Gamma(\alpha)}{ }_{2} F_{1}\left(\alpha+\beta,-\eta ; \alpha ; 1-\frac{\tau}{t}\right) \\
&(\tau \in(0, t) ; t>0) \\
&= \frac{1}{\Gamma(\alpha)} \frac{(t-\tau)^{\alpha-1}}{t^{\alpha+\beta}}+\frac{(\alpha+\beta)(-\eta)}{\Gamma(\alpha+1)} \frac{(t-\tau)^{\alpha}}{t^{\alpha+\beta+1}} \\
&+\frac{(\alpha+\beta)(\alpha+\beta+1)(-\eta)(-\eta+1)}{\Gamma(\alpha+2)} \frac{(t-\tau)^{\alpha+1}}{t^{\alpha+\beta+2}}+\cdots .
\end{aligned}
$$

We observe that each term of the above series is nonnegative under the conditions in Lemma 7 , and, hence, the function $F(t, \tau)$ remains nonnegative for all $\tau \in(0, t)(t>0)$.

Now, by multiplying both sides of (35) by $F(t, \tau) u(\tau)$ defined by (36), integrating the resulting inequality with respect to $\tau$ from 0 to $t$, and using (8), we get

$$
\begin{aligned}
& I_{0, t}^{\alpha, \beta, \eta}\{u(t) f(t) g(t)\}+f(\rho) g(\rho) I_{0, t}^{\alpha, \beta, \eta}\{u(t)\} \\
& \quad \geq g(\rho) I_{0, t}^{\alpha, \beta, \eta}\{u(t) f(t)\}+f(\rho) I_{0, t}^{\alpha, \beta, \eta}\{u(t) g(t)\} .
\end{aligned}
$$

Next, by multiplying both sides of (37) by $F(t, \rho) v(\rho)(\rho \epsilon$ $(0, t) ; t>0)$, where $F(t, \rho)$ is given when $\tau$ is replaced by $\rho$ in (36), integrating the resulting inequality with respect to $\rho$ from 0 to $t$, and using (8), we are led to the desired result (33).

Theorem 8. Let $f$ and $g$ be two continuous and synchronous functions on $[0, \infty)$ and let $l, m, n:[0, \infty) \rightarrow[0, \infty)$ be continuous functions. Then the following inequality holds true:

$$
\begin{aligned}
2 I_{0, t}^{\alpha, \beta, \eta}\{l(t)\}\left[I_{0, t}^{\alpha, \beta, \eta}\{m(t)\} I_{0, t}^{\alpha, \beta, \eta}\{n(t) f(t) g(t)\}\right. \\
\left.+I_{0, t}^{\alpha, \beta, \eta}\{n(t)\} I_{0, t}^{\alpha, \beta, \eta}\{m(t) f(t) g(t)\}\right] \\
+2 I_{0, t}^{\alpha, \beta, \eta}\{m(t)\} I_{0, t}^{\alpha, \beta, \eta}\{n(t)\} I_{0, t}^{\alpha, \beta, \eta}\{l(t) f(t) g(t)\}
\end{aligned}
$$

$$
\begin{aligned}
\geq I_{0, t}^{\alpha, \beta, \eta}\{l(t)\}\left[I_{0, t}^{\alpha, \beta, \eta}\{m(t) f(t)\} I_{0, t}^{\alpha, \beta, \eta}\{n(t) g(t)\}\right. \\
\left.+I_{0, t}^{\alpha, \beta, \eta}\{n(t) f(t)\} I_{0, t}^{\alpha, \beta, \eta}\{m(t) g(t)\}\right] \\
+I_{0, t}^{\alpha, \beta, \eta}\{m(t)\}\left[I_{0, t}^{\alpha, \beta, \eta}\{l(t) f(t)\} I_{0, t}^{\alpha, \beta, \eta}\{n(t) g(t)\}\right. \\
\left.+I_{0, t}^{\alpha, \beta, \eta}\{n(t) f(t)\} I_{0, t}^{\alpha, \beta, \eta}\{l(t) g(t)\}\right] \\
+I_{0, t}^{\alpha, \beta, \eta}\{n(t)\}\left[I_{0, t}^{\alpha, \beta, \eta}\{l(t) f(t)\} I_{0, t}^{\alpha, \beta, \eta}\{m(t) g(t)\}\right. \\
\left.+I_{0, t}^{\alpha, \beta, \eta}\{m(t) f(t)\} I_{0, t}^{\alpha, \beta, \eta}\{l(t) g(t)\}\right],
\end{aligned}
$$

for all $t>0, \alpha>0$, and $\beta, \eta \in \mathbb{R}$ with $\alpha+\beta \geqq 0$ and $\eta \leqq 0$.

Proof. By setting $u=m$ and $v=n$ in Lemma 7, we get

$$
\begin{aligned}
I_{0, t}^{\alpha, \beta, \eta}\{m(t)\} I_{0, t}^{\alpha, \beta, \eta}\{n(t) f(t) g(t)\} \\
+I_{0, t}^{\alpha, \beta, \eta}\{n(t)\} I_{0, t}^{\alpha, \beta, \eta}\{m(t) f(t) g(t)\} \\
\geq I_{0, t}^{\alpha, \beta, \eta}\{m(t) f(t)\} I_{0, t}^{\alpha, \beta, \eta}\{n(t) g(t)\} \\
\quad+I_{0, t}^{\alpha, \beta, \eta}\{n(t) f(t)\} I_{0, t}^{\alpha, \beta, \eta}\{m(t) g(t)\} .
\end{aligned}
$$

Since $I_{0, t}^{\alpha, \beta, \eta}\{l(t)\} \geqq 0$ under the given conditions, by multiplying both sides of (39) by $I_{0, t}^{\alpha, \beta, \eta}\{l(t)\}$, we have

$$
\begin{gathered}
I_{0, t}^{\alpha, \beta, \eta}\{l(t)\}\left[I_{0, t}^{\alpha, \beta, \eta}\{m(t)\} I_{0, t}^{\alpha, \beta, \eta}\{n(t) f(t) g(t)\}\right. \\
\left.+I_{0, t}^{\alpha, \beta, \eta}\{n(t)\} I_{0, t}^{\alpha, \beta, \eta}\{m(t) f(t) g(t)\}\right] \\
\geq I_{0, t}^{\alpha, \beta, \eta}\{l(t)\}\left[I_{0, t}^{\alpha, \beta, \eta}\{m(t) f(t)\} I_{0, t}^{\alpha, \beta, \eta}\{n(t) g(t)\}\right. \\
\left.+I_{0, t}^{\alpha, \beta, \eta}\{n(t) f(t)\} I_{0, t}^{\alpha, \beta, \eta}\{m(t) g(t)\}\right] .
\end{gathered}
$$

Similarly, by replacing $u, v$ by $l, n$ and $u, v$ by $l, m$, respectively, in (33) and then multiplying both sides of the resulting inequalities by $I_{0, t}^{\alpha, \beta, \eta}\{m(t)\}$ and $I_{0, t}^{\alpha, \beta, \eta}\{n(t)\}$ both of which are nonnegative under the given assumptions, respectively, we get the following inequalities:

$$
\begin{gathered}
I_{0, t}^{\alpha, \beta, \eta}\{m(t)\}\left[I_{0, t}^{\alpha, \beta, \eta}\{l(t)\} I_{0, t}^{\alpha, \beta, \eta}\{n(t) f(t) g(t)\}\right. \\
\left.+I_{0, t}^{\alpha, \beta, \eta}\{n(t)\} I_{0, t}^{\alpha, \beta, \eta}\{l(t) f(t) g(t)\}\right] \\
\geq I_{0, t}^{\alpha, \beta, \eta}\{m(t)\}\left[I_{0, t}^{\alpha, \beta, \eta}\{l(t) f(t)\} I_{0, t}^{\alpha, \beta, \eta}\{n(t) g(t)\}\right. \\
\left.+I_{0, t}^{\alpha, \beta, \eta}\{n(t) f(t)\} I_{0, t}^{\alpha, \beta, \eta}\{l(t) g(t)\}\right],
\end{gathered}
$$




$$
\begin{array}{r}
I_{0, t}^{\alpha, \beta, \eta}\{n(t)\}\left[I_{0, t}^{\alpha, \beta, \eta}\{l(t)\} I_{0, t}^{\alpha, \beta, \eta}\{m(t) f(t) g(t)\}\right. \\
\left.+I_{0, t}^{\alpha, \beta, \eta}\{m(t)\} I_{0, t}^{\alpha, \beta, \eta}\{l(t) f(t) g(t)\}\right] \\
\geq I_{0, t}^{\alpha, \beta, \eta}\{n(t)\}\left[I_{0, t}^{\alpha, \beta, \eta}\{l(t) f(t)\} I_{0, t}^{\alpha, \beta, \eta}\{m(t) g(t)\}\right. \\
\left.+I_{0, t}^{\alpha, \beta, \eta}\{m(t) f(t)\} I_{0, t}^{\alpha, \beta, \eta}\{l(t) g(t)\}\right] .
\end{array}
$$

Finally, by adding (40) and (41), side by side, we arrive at the desired result (38).

We present another inequality involving the Saigo fractional integral operator in (8) asserted by the following lemma.

Lemma 9. Let $f$ and $g$ be two continuous and synchronous functions on $[0, \infty)$ and let $u, v:[0, \infty) \rightarrow[0, \infty)$ be continuous functions. Then the following inequality holds true:

$$
\begin{aligned}
I_{0, t}^{\gamma, \delta, \zeta}\{ & v(t)\} I_{0, t}^{\alpha, \beta, \eta}\{u(t) f(t) g(t)\} \\
& +I_{0, t}^{\gamma, \delta, \zeta}\{v(t) f(t) g(t)\} I_{0, t}^{\alpha, \beta, \eta}\{u(t)\} \\
\geq & I_{0, t}^{\gamma, \delta, \zeta}\{v(t) g(t)\} I_{0, t}^{\alpha, \beta, \eta}\{u(t) f(t)\} \\
& +I_{0, t}^{\gamma, \delta, \zeta}\{v(t) f(t)\} I_{0, t}^{\alpha, \beta, \eta}\{u(t) g(t)\},
\end{aligned}
$$

for all $t>0, \alpha>0, \gamma>0$, and $\beta, \eta, \delta, \zeta \in \mathbb{R}$ with $\alpha+\beta \geqq 0$, $\eta \leqq 0, \gamma+\delta \geqq 0$, and $\zeta \leqq 0$.

Proof. By multiplying both sides of (37) by

$$
\begin{array}{r}
\frac{t^{-\gamma-\delta}(t-\rho)^{\gamma-1}}{\Gamma(\gamma)}{ }_{2} F_{1}\left(\gamma+\delta,-\zeta ; \gamma ; 1-\frac{\rho}{t}\right) v(\rho) \\
(\rho \in(0, t) ; t>0),
\end{array}
$$

which remains nonnegative under the conditions in (42), integrating the resulting inequality with respect to $\rho$ from 0 to $t$, and using (8), we get the desired result (42).

Theorem 10. Let $f$ and $g$ be two continuous and synchronous functions on $[0, \infty)$ and let $l, m, n:[0, \infty) \rightarrow[0, \infty)$ be continuous functions. Then the following inequality holds true:

$$
\begin{array}{r}
I_{0, t}^{\alpha, \beta, \eta}\{l(t)\}\left[2 I_{0, t}^{\alpha, \beta, \eta}\{m(t)\} I_{0, t}^{\gamma, \delta, \zeta}\{n(t) f(t) g(t)\}\right. \\
+I_{0, t}^{\alpha, \beta, \eta}\{n(t)\} I_{0, t}^{\gamma, \delta, \zeta}\{m(t) f(t) g(t)\} \\
\left.+I_{0, t}^{\gamma, \delta, \zeta}\{n(t)\} I_{0, t}^{\alpha, \beta, \eta}\{m(t) f(t) g(t)\}\right] \\
+I_{0, t}^{\alpha, \beta, \eta}\{l(t) f(t) g(t)\}\left[I_{0, t}^{\alpha, \beta, \eta}\{m(t)\} I_{0, t}^{\gamma, \delta, \zeta}\{n(t)\}\right. \\
\left.+I_{0, t}^{\alpha, \beta, \eta}\{n(t)\} I_{0, t}^{\gamma, \delta, \zeta}\{m(t)\}\right]
\end{array}
$$

$$
\begin{array}{r}
\geq I_{0, t}^{\alpha, \beta, \eta}\{l(t)\}\left[I_{0, t}^{\alpha, \beta, \eta}\{m(t) f(t)\} I_{0, t}^{\gamma, \delta, \zeta}\{n(t) g(t)\}\right. \\
\left.+I_{0, t}^{\alpha, \beta, \eta}\{m(t) g(t)\} I_{0, t}^{\gamma, \delta, \zeta}\{n(t) f(t)\}\right] \\
+I_{0, t}^{\alpha, \beta, \eta}\{m(t)\}\left[I_{0, t}^{\alpha, \beta, \eta}\{l(t) f(t)\} I_{0, t}^{\gamma, \delta, \zeta}\{n(t) g(t)\}\right. \\
\left.+I_{0, t}^{\alpha, \beta, \eta}\{l(t) g(t)\} I_{0, t}^{\gamma, \delta, \zeta}\{n(t) f(t)\}\right] \\
+I_{0, t}^{\alpha, \beta, \eta}\{n(t)\}\left[I_{0, t}^{\alpha, \beta, \eta}\{l(t) f(t)\} I_{0, t}^{\gamma, \delta, \zeta}\{m(t) g(t)\}\right. \\
\left.+I_{0, t}^{\alpha, \beta, \eta}\{l(t) g(t)\} I_{0, t}^{\gamma, \delta, \zeta}\{m(t) f(t)\}\right],
\end{array}
$$

for all $t>0, \alpha>0, \gamma>0$, and $\beta, \eta, \delta, \zeta \in \mathbb{R}$ with $\alpha+\beta \geqq 0$, $\eta \leqq 0, \gamma+\delta \geqq 0$, and $\zeta \leqq 0$.

Proof. By setting $u=m$ and $v=n$ in (42), we have

$$
\begin{aligned}
I_{0, t}^{\gamma, \delta, \zeta}\{n(t)\} I_{0, t}^{\alpha, \beta, \eta}\{m(t) f(t) g(t)\} \\
+I_{0, t}^{\gamma, \delta, \zeta}\{n(t) f(t) g(t)\} I_{0, t}^{\alpha, \beta, \eta}\{m(t)\} \\
\geq I_{0, t}^{\gamma, \delta, \zeta}\{n(t) g(t)\} I_{0, t}^{\alpha, \beta, \eta}\{m(t) f(t)\} \\
\quad+I_{0, t}^{\gamma, \delta, \zeta}\{n(t) f(t)\} I_{0, t}^{\alpha, \beta, \eta}\{m(t) g(t)\} .
\end{aligned}
$$

By multiplying both sides of (45) by $I_{0, t}^{\alpha, \beta, \eta}\{l(t)\}$, after a little simplification, we get

$$
\begin{array}{r}
I_{0, t}^{\alpha, \beta, \eta}\{l(t)\}\left[I_{0, t}^{\gamma, \delta, \zeta}\{n(t)\} I_{0, t}^{\alpha, \beta, \eta}\{m(t) f(t) g(t)\}\right. \\
\left.+I_{0, t}^{\gamma, \delta, \zeta}\{n(t) f(t) g(t)\} I_{0, t}^{\alpha, \beta, \eta}\{m(t)\}\right] \\
\geq I_{0, t}^{\alpha, \beta, \eta}\{l(t)\}\left[I_{0, t}^{\gamma, \delta, \zeta}\{n(t) g(t)\} I_{0, t}^{\alpha, \beta, \eta}\{m(t) f(t)\}\right. \\
\left.+I_{0, t}^{\gamma, \delta, \zeta}\{n(t) f(t)\} I_{0, t}^{\alpha, \beta, \eta}\{m(t) g(t)\}\right] .
\end{array}
$$

Now, by replacing $u, v$ by $l, n$ and $u, v$ by $l, m$ in (42), respectively, and then multiplying both sides of the resulting inequalities by $I_{0, t}^{\alpha, \beta, \eta}\{m(t)\}$ and $I_{0, t}^{\alpha, \beta, \eta}\{n(t)\}$, respectively, we get the following two inequalities:

$$
\begin{array}{r}
I_{0, t}^{\alpha, \beta, \eta}\{m(t)\}\left[I_{0, t}^{\gamma, \delta, \zeta}\{n(t)\} I_{0, t}^{\alpha, \beta, \eta}\{l(t) f(t) g(t)\}\right. \\
\left.+I_{0, t}^{\gamma, \delta, \zeta}\{n(t) f(t) g(t)\} I_{0, t}^{\alpha, \beta, \eta}\{l(t)\}\right] \\
\geq I_{0, t}^{\alpha, \beta, \eta}\{m(t)\}\left[I_{0, t}^{\gamma, \delta, \zeta}\{n(t) g(t)\} I_{0, t}^{\alpha, \beta, \eta}\{l(t) f(t)\}\right. \\
\left.+I_{0, t}^{\gamma, \delta, \zeta}\{n(t) f(t)\} I_{0, t}^{\alpha, \beta, \eta}\{l(t) g(t)\}\right],
\end{array}
$$




$$
\begin{array}{r}
I_{0, t}^{\alpha, \beta, \eta}\{n(t)\}\left[I_{0, t}^{\gamma, \delta, \zeta}\{m(t)\} I_{0, t}^{\alpha, \beta, \eta}\{l(t) f(t) g(t)\}\right. \\
\left.+I_{0, t}^{\gamma, \delta, \zeta}\{m(t) f(t) g(t)\} I_{0, t}^{\alpha, \beta, \eta}\{l(t)\}\right] \\
\geq I_{0, t}^{\alpha, \beta, \eta}\{n(t)\}\left[I_{0, t}^{\gamma, \delta, \zeta}\{m(t) g(t)\} I_{0, t}^{\alpha, \beta, \eta}\{l(t) f(t)\}\right. \\
\left.+I_{0, t}^{\gamma, \delta, \zeta}\{m(t) f(t)\} I_{0, t}^{\alpha, \beta, \eta}\{l(t) g(t)\}\right] .
\end{array}
$$

Finally, we find that the inequality (44) follows by adding the inequalities (46) and (47), side by side.

Remark 11. It may be noted that the inequalities (38) and (44) in Theorems 8 and 10, respectively, are reversed, if the functions are asynchronous on $[0, \infty)$. The special case of (44) in Theorem 10, when $\alpha=\gamma, \beta=\delta$, and $\eta=\zeta$, is easily seen to yield the inequality (38) in Theorem 8 .

Here, we derive certain, presumably, new integral inequalities by setting $\beta=0$ in (38) and $\beta=0=\delta$ in (44), respectively, and applying the integral operator (12) to the resulting inequalities, we obtain two integral inequalities involving Erdélyi-Kober fractional integral operators stated in Corollaries 12 and 13 below.

Corollary 12. Let $f$ and $g$ be two continuous and synchronous functions on $[0, \infty)$ and let $l, m, n:[0, \infty) \rightarrow[0, \infty)$ be continuous functions. Then the following inequality holds true:

$$
\begin{gathered}
2 I_{0, t}^{\alpha, \eta}\{l(t)\}\left[I_{0, t}^{\alpha, \eta}\{m(t)\} I_{0, t}^{\alpha, \eta}\{n(t) f(t) g(t)\}\right. \\
\left.+I_{0, t}^{\alpha, \eta}\{n(t)\} I_{0, t}^{\alpha, \eta}\{m(t) f(t) g(t)\}\right] \\
+2 I_{0, t}^{\alpha, \eta}\{m(t)\} I_{0, t}^{\alpha, \eta}\{n(t)\} I_{0, t}^{\alpha, \eta}\{l(t) f(t) g(t)\} \\
\geq I_{0, t}^{\alpha, \eta}\{l(t)\}\left[I_{0, t}^{\alpha, \eta}\{m(t) f(t)\} I_{0, t}^{\alpha, \eta}\{n(t) g(t)\}\right. \\
\left.+I_{0, t}^{\alpha, \eta}\{n(t) f(t)\} I_{0, t}^{\alpha, \eta}\{m(t) g(t)\}\right] \\
+I_{0, t}^{\alpha, \eta}\{m(t)\}\left[I_{0, t}^{\alpha, \eta}\{l(t) f(t)\} I_{0, t}^{\alpha, \eta}\{n(t) g(t)\}\right. \\
\left.+I_{0, t}^{\alpha, \eta}\{n(t) f(t)\} I_{0, t}^{\alpha, \eta}\{l(t) g(t)\}\right] \\
+I_{0, t}^{\alpha, \eta}\{n(t)\}\left[I_{0, t}^{\alpha, \eta}\{l(t) f(t)\} I_{0, t}^{\alpha, \eta}\{m(t) g(t)\}\right. \\
\left.+I_{0, t}^{\alpha, \eta}\{m(t) f(t)\} I_{0, t}^{\alpha, \eta}\{l(t) g(t)\}\right]
\end{gathered}
$$

for all $t>0, \alpha>0$, and $\eta \in \mathbb{R}$.
Corollary 13. Let $f$ and $g$ be two continuous and synchronous functions on $[0, \infty)$ and let $l, m, n:[0, \infty) \rightarrow[0, \infty)$ be continuous functions. Then the following inequality holds true:

$$
\begin{gathered}
I_{0, t}^{\alpha, \eta}\{l(t)\}\left[2 I_{0, t}^{\alpha, \eta}\{m(t)\} I_{0, t}^{\gamma, \zeta}\{n(t) f(t) g(t)\}\right. \\
+I_{0, t}^{\alpha, \eta}\{n(t)\} I_{0, t}^{\gamma, \zeta}\{m(t) f(t) g(t)\} \\
\left.+I_{0, t}^{\gamma, \zeta}\{n(t)\} I_{0, t}^{\alpha, \eta}\{m(t) f(t) g(t)\}\right] \\
+I_{0, t}^{\alpha, \eta}\{l(t) f(t) g(t)\}\left[I_{0, t}^{\alpha, \eta}\{m(t)\} I_{0, t}^{\gamma, \zeta}\{n(t)\}\right. \\
\left.+\quad I_{0, t}^{\alpha, \eta}\{n(t)\} I_{0, t}^{\gamma, \zeta}\{m(t)\}\right] \\
\geq I_{0, t}^{\alpha, \eta}\{l(t)\}\left[I_{0, t}^{\alpha, \eta}\{m(t) f(t)\} I_{0, t}^{\gamma, \zeta}\{n(t) g(t)\}\right. \\
\left.+I_{0, t}^{\alpha, \eta}\{m(t) g(t)\} I_{0, t}^{\gamma, \zeta}\{n(t) f(t)\}\right] \\
+I_{0, t}^{\alpha, \eta}\{m(t)\}\left[I_{0, t}^{\alpha, \eta}\{l(t) f(t)\} I_{0, t}^{\gamma, \zeta}\{n(t) g(t)\}\right. \\
\left.+I_{0, t}^{\alpha, \eta}\{l(t) g(t)\} I_{0, t}^{\gamma, \zeta}\{n(t) f(t)\}\right] \\
\left.+I_{0, t}^{\alpha, \eta}\{l(t) g(t)\} I_{0, t}^{\gamma, \zeta}\{m(t) f(t)\}\right] \\
+I_{0, t}^{\alpha, \eta}\{n(t)\}\left[I_{0, t}^{\alpha, \eta}\{l(t) f(t)\} I_{0, t}^{\gamma, \zeta}\{m(t) g(t)\}\right.
\end{gathered}
$$

for all $t>0, \alpha>0, \gamma>0$, and $\eta, \zeta \in \mathbb{R}$.

Remark 14. The special cases $\beta=-\alpha$ in Theorem 8 and $\beta=$ $-\alpha$ and $\delta=-\gamma$ in Theorem 10 are seen to yield the known fractional integral inequalities due to Dahmani [21].

\section{Saigo Fractional $q$-Integral Inequalities}

We establish certain $q$-integral inequalities which are the $q$ analogues (or extensions) of the results derived in the preceding section, some of which are presumably new ones. For our purpose, we begin with providing comparison properties for the fractional $q$-integral operators asserted by the following lemma.

Lemma 15. Let $0<q<1$ and $f:[0, \infty) \rightarrow \mathbb{R}$ be continuous functions with $f(t) \geq 0$, for all $t \in[0, \infty)$. Then we have the following inequalities:

(i) the Saigo fractional q-integral operator of the function $f(t)$ in (30):

$$
I_{q}^{\alpha, \beta, \eta}\{f(t)\} \geq 0
$$

for all $\alpha>0$ and $\beta, \eta \in \mathbb{R}$ with $\alpha+\beta>0$ and $\eta<0$;

(ii) the q-analogue of Riemann-Liouville fractional integral operator of the function $f(t)$ of an order $\alpha$ in (31):

$$
I_{q}^{\alpha}\{f(t)\} \geq 0
$$

for all $\alpha>0$; 
(iii) the q-analogue of Erdélyi-Kober fractional integral operator of the function $f(t)$ in (32):

$$
I_{q}^{\eta, \alpha}\{f(t)\} \geq 0
$$

for all $\alpha>0$ and $\eta \in \mathbb{R}$.

Proof. By applying (23) to the q-integral in (30), we have

$$
\begin{aligned}
I_{q}^{\alpha, \beta, \eta}\{f(t)\}= & \frac{t^{-\beta-1}}{\Gamma_{q}(\alpha)} \\
& \times \sum_{m=0}^{\infty} \frac{\left(q^{\alpha+\beta} ; q\right)_{m}\left(q^{-\eta} ; q\right)_{m}}{\left(q^{\alpha} ; q\right)_{m}(q ; q)_{m}} q^{(\eta-\beta) m}(-1)^{m} q^{-\left(\begin{array}{c}
m \\
2
\end{array}\right)} \\
& \cdot(1-q) t \sum_{j=0}^{\infty} q^{j}\left\{\left(q^{j+1} ; q\right)_{\alpha-1}\left(q^{j}-1\right)_{q}^{m} f\left(q^{j} t\right)\right\} .
\end{aligned}
$$

It is easy to see that

$$
\begin{gathered}
\Gamma_{q}(\alpha)>0 ; \quad\left(q^{\alpha} ; q\right)_{m}>0 ; \\
\left(q^{j+1} ; q\right)_{\alpha-1}=\frac{\left(q^{j+1} ; q\right)_{\infty}}{\left(q^{\alpha+j} ; q\right)_{\infty}}>0
\end{gathered}
$$

for all $\alpha>0$ and $j, m \in \mathbb{N}_{0}$. Next, for simplicity,

$$
h(j, m ; q):=(-1)^{m}\left(q^{j}-1\right)_{q}^{m} \quad\left(j, m \in \mathbb{N}_{0}\right) .
$$

Then we claim that $h(j, m ; q) \geq 0$, for all $j, m \in \mathbb{N}_{0}$. We find from (29) that

$$
\begin{aligned}
h(j, m ; q) & =(-1)^{m} q^{j m}\left(\frac{1}{q^{j}} ; q\right)_{m} \\
& =q^{j m}(-1)^{m} \prod_{k=0}^{m-1}\left(1-q^{k-j}\right) .
\end{aligned}
$$

It is easy to see that, if $m>j$, then $h(j, m ; q)=0$. On the other hand, if $m \leq j$, then we have

$$
h(j, m ; q)=q^{j m} \prod_{k=0}^{m-1}\left(q^{k-j}-1\right)>0,
$$

since $k-j<0$, for all $k$ with $0 \leq k \leq m-1<m \leq j$. Finally, we find that, under the given conditions, each term in the double series of (53) is nonnegative. This completes the proof of (50). The other two inequalities in (51) and (52) may be easily proved.
For convenience and simplicity, we define the following function $\mathscr{H}$ by

$$
\begin{aligned}
& \mathscr{H}(\tau, t, u(\tau) ; \alpha, \beta, \eta ; q) \\
& :=\frac{t^{-\beta-1}}{\Gamma_{q}(\alpha)}\left(\frac{q \tau}{t} ; q\right)_{\alpha-1} \\
& \quad \cdot \sum_{m=0}^{\infty} \frac{\left(q^{\alpha+\beta} ; q\right)_{m}\left(q^{-\eta} ; q\right)_{m}}{\left(q^{\alpha} ; q\right)_{m}(q ; q)_{m}} \\
& \quad \times q^{(\eta-\beta) m}(-1)^{m} q^{-\left(\begin{array}{c}
m \\
2
\end{array}\right)\left(\frac{\tau}{t}-1\right)_{q}^{m} u(\tau),}
\end{aligned}
$$

where $t>0,0 \leq \tau \leq t ; \alpha>0, \beta, \eta \in \mathbb{R}$ with $\alpha+\beta>0$ and $\eta<0 ; 0<q<1 ; u:[0, \infty) \rightarrow[0, \infty)$ is a continuous function. As in the process of Lemma 15 , it is seen that

$$
\mathscr{H}(\tau, t, u(\tau) ; \alpha, \beta, \eta ; q) \geq 0,
$$

under the conditions given in (58).

Here, we present two $q$-integral inequalities involving the Saigo fractional $q$-integral operator (30) stated in Lemmas 16 and 17 below.

Lemma 16. Let $0<q<1$; let $f$ and $g$ be two continuous and synchronous functions on $[0, \infty)$; let $u:[0, \infty) \rightarrow[0, \infty)$ be a continuous function. Then the following inequality holds true, for $t \in(0, \infty)$,

$$
\begin{aligned}
I_{q}^{\alpha, \beta, \eta} & \{u(t)\} I_{q}^{\alpha, \beta, \eta}\{v(t) f(t) g(t)\} \\
& +I_{q}^{\alpha, \beta, \eta}\{v(t)\} I_{q}^{\alpha, \beta, \eta}\{u(t) f(t) g(t)\} \\
\geq & I_{q}^{\alpha, \beta, \eta}\{u(t) f(t)\} I_{q}^{\alpha, \beta, \eta}\{v(t) g(t)\} \\
& +I_{q}^{\alpha, \beta, \eta}\{v(t) f(t)\} I_{q}^{\alpha, \beta, \eta}\{u(t) g(t)\},
\end{aligned}
$$

for all $\alpha>0$ and $\beta, \eta \in \mathbb{R}$ with $\alpha+\beta>0$ and $\eta<0$.

Proof. Since $f$ and $g$ are two synchronous functions on $[0, \infty)$, for all $\tau, \rho \geq 0$, the inequality (35) is satisfied. By multiplying both sides of (35) by $\mathscr{H}(\tau, t, u(\tau) ; \alpha, \beta, \eta ; q)$ in (58) together with (59) and taking $q$-integration of the resulting inequality with respect to $\tau$ from 0 to $t$ with the aid of Definition 4, we get

$$
\begin{aligned}
& I_{q}^{\alpha, \beta, \eta}\{u(t) f(t) g(t)\}+f(\rho) g(\rho) I_{q}^{\alpha, \beta, \eta}\{u(t)\} \\
& \quad \geq g(\rho) I_{q}^{\alpha, \beta, \eta}\{u(t) f(t)\}+f(\rho) I_{q}^{\alpha, \beta, \eta}\{u(t) g(t)\} .
\end{aligned}
$$

Next, by multiplying both sides of (61) by $\mathscr{H}(\rho, t, v(\rho) ; \alpha, \beta, \eta ; q)$ in (58) together with (59), taking $q$-integration of the last resulting inequality with respect to $\rho$ from 0 to $t$, and using Definition 4 , we are led to the desired result (60).

Lemma 17. Let $0<q<1$; let $f$ and $g$ be two continuous and synchronous functions on $[0, \infty)$; let $u, v:[0, \infty) \rightarrow[0, \infty)$ 
be continuous functions. Then the following inequality holds true, for $t \in(0, \infty)$,

$$
\begin{aligned}
I_{q}^{\gamma, \delta, \zeta}\{v(t)\} I_{q}^{\alpha, \beta, \eta}\{u(t) f(t) g(t)\} \\
+I_{q}^{\gamma, \delta, \zeta}\{v(t) f(t) g(t)\} I_{q}^{\alpha, \beta, \eta}\{u(t)\} \\
\geq I_{q}^{\gamma, \delta, \zeta}\{v(t) g(t)\} I_{q}^{\alpha, \beta, \eta}\{u(t) f(t)\} \\
\quad+I_{q}^{\gamma, \delta, \zeta}\{v(t) f(t)\} I_{q}^{\alpha, \beta, \eta}\{u(t) g(t)\},
\end{aligned}
$$

for all $\alpha>0, \gamma>0$, and $\beta, \eta, \delta, \zeta \in \mathbb{R}$ with $\alpha+\beta>0, \gamma+\delta>0$, $\eta<0$, and $\zeta<0$.

Proof. By multiplying both sides of (61) by $\mathscr{H}(\rho, t, v(\rho) ; \gamma, \delta, \zeta ; q)$ in (58) together with (59) and taking the $q$-integration of the resulting inequality with respect to $\rho$ from 0 to $t$ with the aid of Definition 4 , we get the desired result (62).

Theorem 18. Let $0<q<1$; let $f, g:[0, \infty) \rightarrow \mathbb{R}$ be two continuous synchronous functions; let $l, m, n:[0, \infty) \rightarrow$ $[0, \infty)$ be continuous functions. Then the following inequality holds true, fort $\in(0, \infty)$,

$$
\begin{array}{r}
2 I_{q}^{\alpha, \beta, \eta}\{l(t)\}\left[I_{q}^{\alpha, \beta, \eta}\{m(t)\} I_{q}^{\alpha, \beta, \eta}\{n(t) f(t) g(t)\}\right. \\
\left.+I_{q}^{\alpha, \beta, \eta}\{n(t)\} I_{q}^{\alpha, \beta, \eta}\{m(t) f(t) g(t)\}\right] \\
+2 I_{q}^{\alpha, \beta, \eta}\{m(t)\} I_{q}^{\alpha, \beta, \eta}\{n(t)\} I_{q}^{\alpha, \beta, \eta}\{l(t) f(t) g(t)\} \\
\geq I_{q}^{\alpha, \beta, \eta}\{l(t)\}\left[I_{q}^{\alpha, \beta, \eta}\{m(t) f(t)\} I_{q}^{\alpha, \beta, \eta}\{n(t) g(t)\}\right. \\
\left.+I_{q}^{\alpha, \beta, \eta}\{n(t) f(t)\} I_{q}^{\alpha, \beta, \eta}\{m(t) g(t)\}\right] \\
+I_{q}^{\alpha, \beta, \eta}\{m(t)\}\left[I_{q}^{\alpha, \beta, \eta}\{l(t) f(t)\} I_{q}^{\alpha, \beta, \eta}\{n(t) g(t)\}\right. \\
\left.+I_{q}^{\alpha, \beta, \eta}\{n(t) f(t)\} I_{q}^{\alpha, \beta, \eta}\{l(t) g(t)\}\right] \\
+I_{q}^{\alpha, \beta, \eta}\{n(t)\}\left[I_{q}^{\alpha, \beta, \eta}\{l(t) f(t)\} I_{q}^{\alpha, \beta, \eta}\{m(t) g(t)\}\right. \\
\left.+I_{q}^{\alpha, \beta, \eta}\{m(t) f(t)\} I_{q}^{\alpha, \beta, \eta}\{l(t) g(t)\}\right]
\end{array}
$$

for all $\alpha>0$ and $\beta, \eta \in \mathbb{R}$ with $\alpha+\beta>0$ and $\eta<0$.

Proof. We start with (60) in Lemma 16; by putting $u=m$ and $v=n$, we get

$$
\begin{aligned}
I_{q}^{\alpha, \beta, \eta} & \{m(t)\} I_{q}^{\alpha, \beta, \eta}\{n(t) f(t) g(t)\} \\
& +I_{q}^{\alpha, \beta, \eta}\{n(t)\} I_{q}^{\alpha, \beta, \eta}\{m(t) f(t) g(t)\} \\
\geq & I_{q}^{\alpha, \beta, \eta}\{m(t) f(t)\} I_{q}^{\alpha, \beta, \eta}\{n(t) g(t)\} \\
& +I_{q}^{\alpha, \beta, \eta}\{n(t) f(t)\} I_{q}^{\alpha, \beta, \eta}\{m(t) g(t)\} .
\end{aligned}
$$

By multiplying both sides of (64) by $I_{q}^{\alpha, \beta, \eta}\{l(t)\}$, we have

$$
\begin{aligned}
I_{q}^{\alpha, \beta, \eta}\{l(t)\}\left[I_{q}^{\alpha, \beta, \eta}\{m(t)\} I_{q}^{\alpha, \beta, \eta}\{n(t) f(t) g(t)\}\right. \\
\left.+I_{q}^{\alpha, \beta, \eta}\{n(t)\} I_{q}^{\alpha, \beta, \eta}\{m(t) f(t) g(t)\}\right] \\
\geq I_{q}^{\alpha, \beta, \eta}\{l(t)\}\left[I_{q}^{\alpha, \beta, \eta}\{m(t) f(t)\} I_{q}^{\alpha, \beta, \eta}\{n(t) g(t)\}\right. \\
\left.+I_{q}^{\alpha, \beta, \eta}\{n(t) f(t)\} I_{q}^{\alpha, \beta, \eta}\{m(t) g(t)\}\right] .
\end{aligned}
$$

Similarly, by replacing $u, v$ by $l, n$ and $u, v$ by $l, m$ in (60), respectively and then multiplying both sides of the resulting inequalities by $I_{q}^{\alpha, \beta, \eta}\{m(t)\}$ and $I_{q}^{\alpha, \beta, \eta}\{n(t)\}$, respectively, we get

$$
\begin{gathered}
I_{q}^{\alpha, \beta, \eta}\{m(t)\}\left[I_{q}^{\alpha, \beta, \eta}\{l(t)\} I_{q}^{\alpha, \beta, \eta}\{n(t) f(t) g(t)\}\right. \\
\left.+I_{q}^{\alpha, \beta, \eta}\{n(t)\} I_{q}^{\alpha, \beta, \eta}\{l(t) f(t) g(t)\}\right] \\
\geq I_{q}^{\alpha, \beta, \eta}\{m(t)\}\left[I_{q}^{\alpha, \beta, \eta}\{l(t) f(t)\} I_{q}^{\alpha, \beta, \eta}\{n(t) g(t)\}\right. \\
\left.+I_{q}^{\alpha, \beta, \eta}\{n(t) f(t)\} I_{q}^{\alpha, \beta, \eta}\{l(t) g(t)\}\right], \\
I_{q}^{\alpha, \beta, \eta}\{n(t)\}\left[I_{q}^{\alpha, \beta, \eta}\{l(t)\} I_{q}^{\alpha, \beta, \eta}\{m(t) f(t) g(t)\}\right. \\
\left.+I_{q}^{\alpha, \beta, \eta}\{m(t)\} I_{q}^{\alpha, \beta, \eta}\{l(t) f(t) g(t)\}\right] \\
\geq I_{q}^{\alpha, \beta, \eta}\{n(t)\}\left[I_{q}^{\alpha, \beta, \eta}\{l(t) f(t)\} I_{q}^{\alpha, \beta, \eta}\{m(t) g(t)\}\right. \\
\left.+I_{q}^{\alpha, \beta, \eta}\{m(t) f(t)\} I_{q}^{\alpha, \beta, \eta}\{l(t) g(t)\}\right] .
\end{gathered}
$$

Finally, by adding (65) and (66), side by side, we arrive at the desired result in Theorem 18.

Theorem 19. Let $0<q<1$; let $f, g:[0, \infty) \rightarrow \mathbb{R}$ be two continuous synchronous functions; let $l, m, n:[0, \infty) \rightarrow$ $[0, \infty)$ be continuous functions. Then the following inequality holds true, for $t \in(0, \infty)$,

$$
\begin{array}{r}
I_{q}^{\alpha, \beta, \eta}\{l(t)\}\left[2 I_{q}^{\alpha, \beta, \eta}\{m(t)\} I_{q}^{\gamma, \delta, \zeta}\{n(t) f(t) g(t)\}\right. \\
+I_{q}^{\alpha, \beta, \eta}\{n(t)\} I_{q}^{\gamma, \delta, \zeta}\{m(t) f(t) g(t)\} \\
\left.+I_{q}^{\gamma, \delta, \zeta}\{n(t)\} I_{q}^{\alpha, \beta, \eta}\{m(t) f(t) g(t)\}\right] \\
+I_{q}^{\alpha, \beta, \eta}\{l(t) f(t) g(t)\}\left[I_{q}^{\alpha, \beta, \eta}\{m(t)\} I_{q}^{\gamma, \delta, \zeta}\{n(t)\}\right. \\
\left.+I_{q}^{\alpha, \beta, \eta}\{n(t)\} I_{q}^{\gamma, \delta, \zeta}\{m(t)\}\right]
\end{array}
$$




$$
\begin{array}{r}
\geq I_{q}^{\alpha, \beta, \eta}\{l(t)\}\left[I_{q}^{\alpha, \beta, \eta}\{m(t) f(t)\} I_{q}^{\gamma, \delta, \zeta}\{n(t) g(t)\}\right. \\
\left.+I_{q}^{\alpha, \beta, \eta}\{m(t) g(t)\} I_{q}^{\gamma, \delta, \zeta}\{n(t) f(t)\}\right] \\
+I_{q}^{\alpha, \beta, \eta}\{m(t)\}\left[I_{q}^{\alpha, \beta, \eta}\{l(t) f(t)\} I_{q}^{\gamma, \delta, \zeta}\{n(t) g(t)\}\right. \\
\left.+I_{q}^{\alpha, \beta, \eta}\{l(t) g(t)\} I_{q}^{\gamma, \delta, \zeta}\{n(t) f(t)\}\right] \\
+I_{q}^{\alpha, \beta, \eta}\{n(t)\}\left[I_{q}^{\alpha, \beta, \eta}\{l(t) f(t)\} I_{q}^{\gamma, \delta, \zeta}\{m(t) g(t)\}\right. \\
\left.+I_{q}^{\alpha, \beta, \eta}\{l(t) g(t)\} I_{q}^{\gamma, \delta, \zeta}\{m(t) f(t)\}\right],
\end{array}
$$

for all $\alpha>0, \gamma>0$, and $\beta, \eta, \delta, \zeta \in \mathbb{R}$ with $\alpha+\beta>0, \gamma+\delta>0$, $\eta<0$, and $\zeta<0$.

Proof. By setting $u=m$ and $v=n$ in (62), we have

$$
\begin{aligned}
& I_{q}^{\gamma, \delta, \zeta}\{n(t)\} I_{q}^{\alpha, \beta, \eta}\{m(t) f(t) g(t)\} \\
&+I_{q}^{\gamma, \delta, \zeta}\{n(t) f(t) g(t)\} I_{q}^{\alpha, \beta, \eta}\{m(t)\} \\
& \geq I_{q}^{\gamma, \delta, \zeta}\{n(t) g(t)\} I_{q}^{\alpha, \beta, \eta}\{m(t) f(t)\} \\
&+ I_{q}^{\gamma, \delta, \zeta}\{n(t) f(t)\} I_{q}^{\alpha, \beta, \eta}\{m(t) g(t)\} .
\end{aligned}
$$

By multiplying both sides of (68) by $I_{q}^{\alpha, \beta, \eta}\{l(t)\}$, after a little simplification, we get

$$
\begin{gathered}
I_{q}^{\alpha, \beta, \eta}\{l(t)\}\left[I_{q}^{\gamma, \delta, \zeta}\{n(t)\} I_{q}^{\alpha, \beta, \eta}\{m(t) f(t) g(t)\}\right. \\
\left.+I_{q}^{\gamma, \delta, \zeta}\{n(t) f(t) g(t)\} I_{q}^{\alpha, \beta, \eta}\{m(t)\}\right] \\
\geq I_{q}^{\alpha, \beta, \eta}\{l(t)\}\left[I_{q}^{\gamma, \delta, \zeta}\{n(t) g(t)\} I_{q}^{\alpha, \beta, \eta}\{m(t) f(t)\}\right. \\
\left.+I_{q}^{\gamma, \delta, \zeta}\{n(t) f(t)\} I_{q}^{\alpha, \beta, \eta}\{m(t) g(t)\}\right] .
\end{gathered}
$$

By replacing $u, v$ by $l, n$ and $u, v$ by $l, m$ in (60) and then multiplying both sides of the resulting inequalities by $I_{q}^{\alpha, \beta, \eta}\{m(t)\}$ and $I_{q}^{\alpha, \beta, \eta}\{n(t)\}$, respectively, we get

$$
\begin{gathered}
I_{q}^{\alpha, \beta, \eta}\{m(t)\}\left[I_{q}^{\gamma, \delta, \zeta}\{n(t)\} I_{q}^{\alpha, \beta, \eta}\{l(t) f(t) g(t)\}\right. \\
\left.+I_{q}^{\gamma, \delta, \zeta}\{n(t) f(t) g(t)\} I_{q}^{\alpha, \beta, \eta}\{l(t)\}\right] \\
\geq I_{q}^{\alpha, \beta, \eta}\{m(t)\}\left[I_{q}^{\gamma, \delta, \zeta}\{n(t) g(t)\} I_{q}^{\alpha, \beta, \eta}\{l(t) f(t)\}\right. \\
\left.+I_{q}^{\gamma, \delta, \zeta}\{n(t) f(t)\} I_{q}^{\alpha, \beta, \eta}\{l(t) g(t)\}\right], \\
I_{q}^{\alpha, \beta, \eta}\{n(t)\}\left[I_{q}^{\gamma, \delta, \zeta}\{m(t)\} I_{q}^{\alpha, \beta, \eta}\{l(t) f(t) g(t)\}\right. \\
\left.+I_{q}^{\gamma, \delta, \zeta}\{m(t) f(t) g(t)\} I_{q}^{\alpha, \beta, \eta}\{l(t)\}\right] \\
\geq I_{q}^{\alpha, \beta, \eta}\{n(t)\}\left[I_{q}^{\gamma, \delta, \zeta}\{m(t) g(t)\} I_{q}^{\alpha, \beta, \eta}\{l(t) f(t)\}\right. \\
\left.+I_{q}^{\gamma, \delta, \zeta}\{m(t) f(t)\} I_{q}^{\alpha, \beta, \eta}\{l(t) g(t)\}\right] .
\end{gathered}
$$

We, therefore, find that the inequality (67) follows by adding the inequalities (69) and (70), side by side.

Remark 20. It is noted that the inequalities in (63) and (67) are reversed if the functions $f$ and $g$ are asynchronous. It is also easily seen that the special cases $\alpha=\gamma, \beta=\delta$, and $\eta=\zeta$ of (67) in Theorem 19 reduce to those in Theorem 18.

Following Garg and Chanchlani [19], the operator (30) would reduce immediately to the extensively investigated $q$-analogue of Erdélyi-Kober and Riemann-Liouville type fractional integral operators in (31) and (32), respectively (see also [20]). Indeed, by suitably specializing the values of parameters $\alpha$ (and additionally $\delta$ in Theorem 19), the results presented in this section would find further fractional $q$ integral inequalities involving the $q$-analogues of ErdélyiKober and Riemann-Liouville type fractional integral operators in (31) and (32). For example, if we set $\beta=-\alpha$ in Theorem 18 and $\beta=-\alpha$ and $\delta=-\gamma$ in Theorem 19, respectively, and make use of the relation (31), we are led to the following, presumably, new $q$-integral inequalities involving $q$-Riemann-Liouville fractional integral operators given in Corollaries 21 and 22 below.

Corollary 21. Let $0<q<1$; let $f, g:[0, \infty) \rightarrow \mathbb{R}$ be two continuous synchronous functions; let $l, m, n:[0, \infty) \rightarrow$ $[0, \infty)$ be continuous functions. Then the following inequality holds true, for $t \in(0, \infty)$,

$$
\begin{gathered}
2 I_{q}^{\alpha}\{l(t)\}\left[I_{q}^{\alpha}\{m(t)\} I_{q}^{\alpha}\{n(t) f(t) g(t)\}\right. \\
\left.+I_{q}^{\alpha}\{n(t)\} I_{q}^{\alpha}\{m(t) f(t) g(t)\}\right] \\
+2 I_{q}^{\alpha}\{m(t)\} I_{q}^{\alpha}\{n(t)\} I_{q}^{\alpha}\{l(t) f(t) g(t)\} \\
\geq I_{q}^{\alpha}\{l(t)\}\left[I_{q}^{\alpha}\{m(t) f(t)\} I_{q}^{\alpha}\{n(t) g(t)\}\right. \\
\left.+I_{q}^{\alpha}\{n(t) f(t)\} I_{q}^{\alpha}\{m(t) g(t)\}\right] \\
+I_{q}^{\alpha}\{m(t)\}\left[I_{q}^{\alpha}\{l(t) f(t)\} I_{q}^{\alpha}\{n(t) g(t)\}\right. \\
\left.+I_{q}^{\alpha}\{n(t) f(t)\} I_{q}^{\alpha}\{l(t) g(t)\}\right] \\
+I_{q}^{\alpha}\{n(t)\}\left[I_{q}^{\alpha}\{l(t) f(t)\} I_{q}^{\alpha}\{m(t) g(t)\}\right. \\
\left.+I_{q}^{\alpha}\{m(t) f(t)\} I_{q}^{\alpha}\{l(t) g(t)\}\right],
\end{gathered}
$$

for all $\alpha>0$.

Corollary 22. Let $0<q<1$; let $f, g:[0, \infty) \rightarrow \mathbb{R}$ be two continuous synchronous functions; let $l, m, n:[0, \infty) \rightarrow$ $[0, \infty)$ be continuous functions. Then the following inequality holds true, for $t \in(0, \infty)$,

$$
\begin{aligned}
I_{q}^{\alpha}\{l(t)\}[ & 2 I_{q}^{\alpha}\{m(t)\} I_{q}^{\gamma}\{n(t) f(t) g(t)\} \\
& +I_{q}^{\alpha}\{n(t)\} I_{q}^{\gamma}\{m(t) f(t) g(t)\}
\end{aligned}
$$




$$
\begin{gathered}
\left.+I_{q}^{\gamma}\{n(t)\} I_{q}^{\alpha}\{m(t) f(t) g(t)\}\right] \\
+I_{q}^{\alpha}\{l(t) f(t) g(t)\}\left[I_{q}^{\alpha}\{m(t)\} I_{q}^{\gamma}\{n(t)\}\right. \\
\left.+I_{q}^{\alpha}\{n(t)\} I_{q}^{\gamma}\{m(t)\}\right] \\
\geq I_{q}^{\alpha}\{l(t)\}\left[I_{q}^{\alpha}\{m(t) f(t)\} I_{q}^{\gamma}\{n(t) g(t)\}\right. \\
\left.+I_{q}^{\alpha}\{m(t) g(t)\} I_{q}^{\gamma}\{n(t) f(t)\}\right] \\
+I_{q}^{\alpha}\{m(t)\}\left[I_{q}^{\alpha}\{l(t) f(t)\} I_{q}^{\gamma}\{n(t) g(t)\}\right. \\
\left.+I_{q}^{\alpha}\{l(t) g(t)\} I_{q}^{\gamma}\{n(t) f(t)\}\right] \\
+I_{q}^{\alpha}\{n(t)\}\left[I_{q}^{\alpha}\{l(t) f(t)\} I_{q}^{\gamma}\{m(t) g(t)\}\right. \\
\left.+I_{q}^{\alpha}\{l(t) g(t)\} I_{q}^{\gamma}\{m(t) f(t)\}\right],
\end{gathered}
$$

for all $\alpha>0$ and $\gamma>0$.

Furthermore, if we set $\beta=0$ in Theorem 18 and $\beta=0=$ $\delta$ in Theorem 19, we obtain the following, presumably, new inequalities involving $q$-analogue of the Erdélyi-Kober type fractional integral operators asserted by Corollaries 23 and 24 below.

Corollary 23. Let $0<q<1$; let $f, g:[0, \infty) \rightarrow \mathbb{R}$ be two continuous synchronous functions; let $l, m, n:[0, \infty) \rightarrow$ $[0, \infty)$ be continuous functions. Then the following inequality holds true, for $t \in(0, \infty)$,

$$
\begin{gathered}
2 I_{q}^{\eta, \alpha}\{l(t)\}\left[I_{q}^{\eta, \alpha}\{m(t)\} I_{q}^{\eta, \alpha}\{n(t) f(t) g(t)\}\right. \\
\left.+I_{q}^{\eta, \alpha}\{n(t)\} I_{q}^{\eta, \alpha}\{m(t) f(t) g(t)\}\right] \\
+2 I_{q}^{\eta, \alpha}\{m(t)\} I_{q}^{\eta, \alpha}\{n(t)\} I_{q}^{\eta, \alpha}\{l(t) f(t) g(t)\} \\
\geq I_{q}^{\eta, \alpha}\{l(t)\}\left[I_{q}^{\eta, \alpha}\{m(t) f(t)\} I_{q}^{\eta, \alpha}\{n(t) g(t)\}\right. \\
\left.+I_{q}^{\eta, \alpha}\{n(t) f(t)\} I_{q}^{\eta, \alpha}\{m(t) g(t)\}\right] \\
+I_{q}^{\eta, \alpha}\{m(t)\}\left[I_{q}^{\eta, \alpha}\{l(t) f(t)\} I_{q}^{\eta, \alpha}\{n(t) g(t)\}\right. \\
\left.+I_{q}^{\eta, \alpha}\{n(t) f(t)\} I_{q}^{\eta, \alpha}\{l(t) g(t)\}\right] \\
+I_{q}^{\eta, \alpha}\{n(t)\}\left[I_{q}^{\eta, \alpha}\{l(t) f(t)\} I_{q}^{\eta, \alpha}\{m(t) g(t)\}\right. \\
\left.+I_{q}^{\eta, \alpha}\{m(t) f(t)\} I_{q}^{\eta, \alpha}\{l(t) g(t)\}\right],
\end{gathered}
$$

for all $\alpha>0$.

Corollary 24. Let $0<q<1$; let $f, g:[0, \infty) \rightarrow \mathbb{R}$ be two continuous synchronous functions; let $l, m, n:[0, \infty) \rightarrow$
$[0, \infty)$ be continuous functions. Then the following inequality holds true, for $t \in(0, \infty)$,

$$
\begin{gathered}
I_{q}^{\eta, \alpha}\{l(t)\}\left[2 I_{q}^{\eta, \alpha}\{m(t)\} I_{q}^{\gamma, \delta}\{n(t) f(t) g(t)\}\right. \\
+I_{q}^{\eta, \alpha}\{n(t)\} I_{q}^{\gamma, \delta}\{m(t) f(t) g(t)\} \\
\left.+I_{q}^{\gamma, \delta}\{n(t)\} I_{q}^{\eta, \alpha}\{m(t) f(t) g(t)\}\right] \\
+I_{q}^{\eta, \alpha}\{l(t) f(t) g(t)\}\left[I_{q}^{\eta, \alpha}\{m(t)\} I_{q}^{\gamma, \delta}\{n(t)\}\right. \\
\left.+I_{q}^{\eta, \alpha}\{n(t)\} I_{q}^{\gamma, \delta}\{m(t)\}\right] \\
\geq I_{q}^{\eta, \alpha}\{l(t)\}\left[I_{q}^{\eta, \alpha}\{m(t) f(t)\} I_{q}^{\gamma, \delta}\{n(t) g(t)\}\right. \\
\left.+I_{q}^{\eta, \alpha}\{m(t) g(t)\} I_{q}^{\gamma, \delta}\{n(t) f(t)\}\right] \\
+I_{q}^{\eta, \alpha}\{m(t)\}\left[I_{q}^{\eta, \alpha}\{l(t) f(t)\} I_{q}^{\gamma}\{n(t) g(t)\}\right. \\
\left.+I_{q}^{\eta, \alpha}\{l(t) g(t)\} I_{q}^{\gamma, \delta}\{n(t) f(t)\}\right] \\
+I_{q}^{\eta, \alpha}\{n(t)\}\left[I_{q}^{\eta, \alpha}\{l(t) f(t)\} I_{q}^{\gamma, \delta}\{m(t) g(t)\}\right. \\
\left.+I_{q}^{\eta, \alpha}\{l(t) g(t)\} I_{q}^{\gamma, \delta}\{m(t) f(t)\}\right],
\end{gathered}
$$

for all $\alpha>0, \delta>0$, and $\gamma, \eta \in \mathbb{R}$.

\section{Concluding Remarks}

It is easy to see that

$$
\begin{aligned}
& \lim _{q \rightarrow 1} \Gamma_{q}(a)=\Gamma(a), \\
& \lim _{q \rightarrow 1} \frac{\left(q^{a} ; q\right)_{n}}{(1-q)^{n}}=(a)_{n},
\end{aligned}
$$

where $(a)_{n}$ is the Pochhammer symbol given in (10). Taking the limit of some of the results presented in Section 3 as $q \rightarrow$ 1 with the aid of (75), the resulting inequalities are seen to correspond with those results in Section 2. It is noted that the inequalities in (71) and (72) are equal to the known ones by Dahmani [21, pp. 494-496, Equations (8) and (24)].

Representations of the inequalities in terms of the fractional integral operators have been investigated by many researchers in the existing literature. Here, we have presented some, presumably new Saigo type fractional integral inequalities and their $q$-analogues by using the one parameters of deformation. Very recently, Baleanu and Agarwal [22] also gave some new Saigo type $q$-fractional integral inequalities by using the two parameters of deformation $q_{1}$ and $q_{2}$, which are totally new and different ones from those presented here.

\section{Conflict of Interests}

The authors declare that there is no conflict of interests regarding the publication of this paper. 


\section{Acknowledgment}

This work was supported by Dongguk University Research Fund of 2013.

\section{References}

[1] J. C. Kuang, Applied Inequalities, Shandong Sciences and Technologie Press, Shandong, China, 2004 (Chinese).

[2] D. S. Mitrinović, Analytic Inequalities, Springer, Berlin, Germany, 1970.

[3] P. L. Chebyshev, "Sur les expressions approximatives des integrales definies par les autres prises entre les mêmes limites," Proceedings of the Mathematical Society of Kharkov, vol. 2, pp. 93-98, 1882.

[4] A. M. Ostrowski, "On an integral inequality," Aequationes Mathematicae, vol. 4, pp. 358-373, 1970.

[5] G. A. Anastassiou, Advances on Fractional Inequalities, Springer Briefs in Mathematics, Springer, New York, NY, USA, 2011.

[6] S. Belarbi and Z. Dahmani, "On some new fractional integral inequalities," Journal of Inequalities in Pure and Applied Mathematics, vol. 10, no. 3, article 86, p. 5, 2009.

[7] Z. Dahmani, O. Mechouar, and S. Brahami, "Certain inequalities related to the Chebyshev's functional involving a RiemannLiouville operator," Bulletin of Mathematical Analysis and Applications, vol. 3, no. 4, pp. 38-44, 2011.

[8] S. S. Dragomir, "Some integral inequalities of Grüss type," Indian Journal of Pure and Applied Mathematics, vol. 31, no. 4, pp. 397-415, 2000.

[9] S. L. Kalla and A. Rao, "On Grüss type inequality for a hypergeometric fractional integral," Le Matematiche, vol. 66, no. 1, pp. 57-64, 2011.

[10] V. Lakshmikantham and A. S. Vatsala, "Theory of fractional differential inequalities and applications," Communications in Applied Analysis, vol. 11, no. 3-4, pp. 395-402, 2007.

[11] H. Ögünmez and U. M. Özkan, "Fractional quantum integral inequalities," Journal of Inequalities and Applications, vol. 2011, Article ID 787939, 7 pages, 2011.

[12] W. T. Sulaiman, "Some new fractional integral inequalities," Journal of Mathematical Analysis, vol. 2, no. 2, pp. 23-28, 2011.

[13] D. Baleanu, S. D. Purohit, and P. Agarwal, "On fractional integral inequalities involving hypergeometric operators," Chinese Journal of Mathematics, vol. 2014, Article ID 609476, 5 pages, 2014.

[14] M. Saigo, "A remark on integral operators involving the Gauss hypergeometric functions," Mathematical Reports of College of General Education, Kyushu University, vol. 11, no. 2, pp. 135-143, 1978.

[15] V. S. Kiryakova, Generalized Fractional Calculus and Applications, vol. 301 of Pitman Research Notes in Mathematical Series, Longman Scientific \& Technical, Harlow, UK, 1994.

[16] H. M. Srivastava and J. Choi, Zeta and q-Zeta Functions and Associated Series and Integrals, Elsevier Science Publishers, New York, NY, USA, 2012.

[17] S. G. Samko, A. A. Kilbas, and O. I. Marichev, Fractional Integrals and Derivatives: Theory and Applications, Gordon and Breach Science Publishers, New York, NY, USA, 1993.

[18] F. H. Jackson, “On $q$-definite integrals," The Quarterly Journal of Pure and Applied Mathematics, vol. 41, pp. 193-203, 1910.
[19] M. Garg and L. Chanchlani, " $q$-analogues of Saigo's fractional calculus operators," Bulletin of Mathematical Analysis and Applications, vol. 3, no. 4, pp. 169-179, 2011.

[20] R. P. Agarwal, "Certain fractional $q$-integrals and $q$-derivatives," Mathematical Proceedings of the Cambridge Philosophical Society, vol. 66, pp. 365-370, 1969.

[21] Z. Dahmani, "New inequalities in fractional integrals," International Journal of Nonlinear Science, vol. 9, no. 4, pp. 493-497, 2010.

[22] D. Baleanu and P. Agarwal, "Certain inequalities involving the fractional $q$-integral operators," In press. 


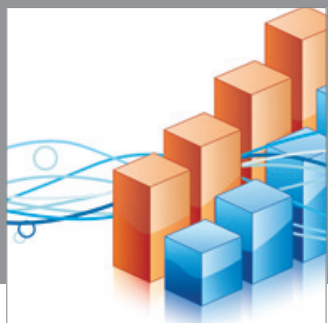

Advances in

Operations Research

mansans

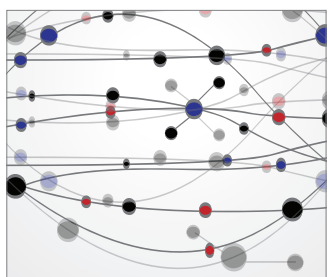

The Scientific World Journal
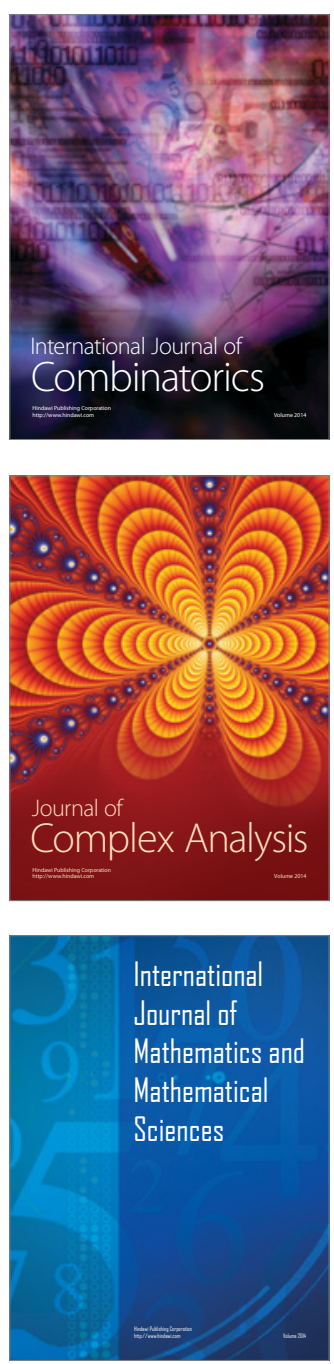
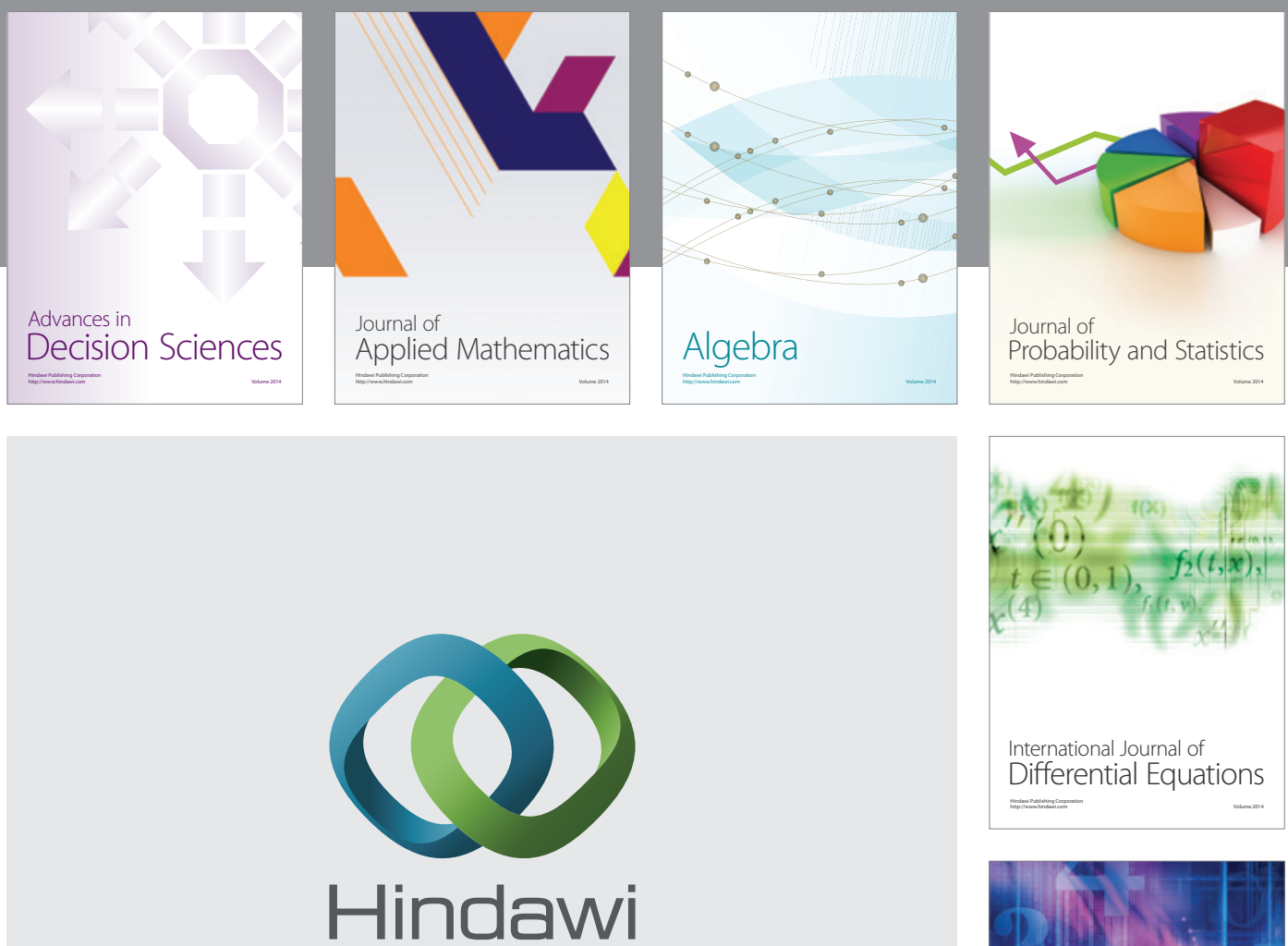

Submit your manuscripts at http://www.hindawi.com
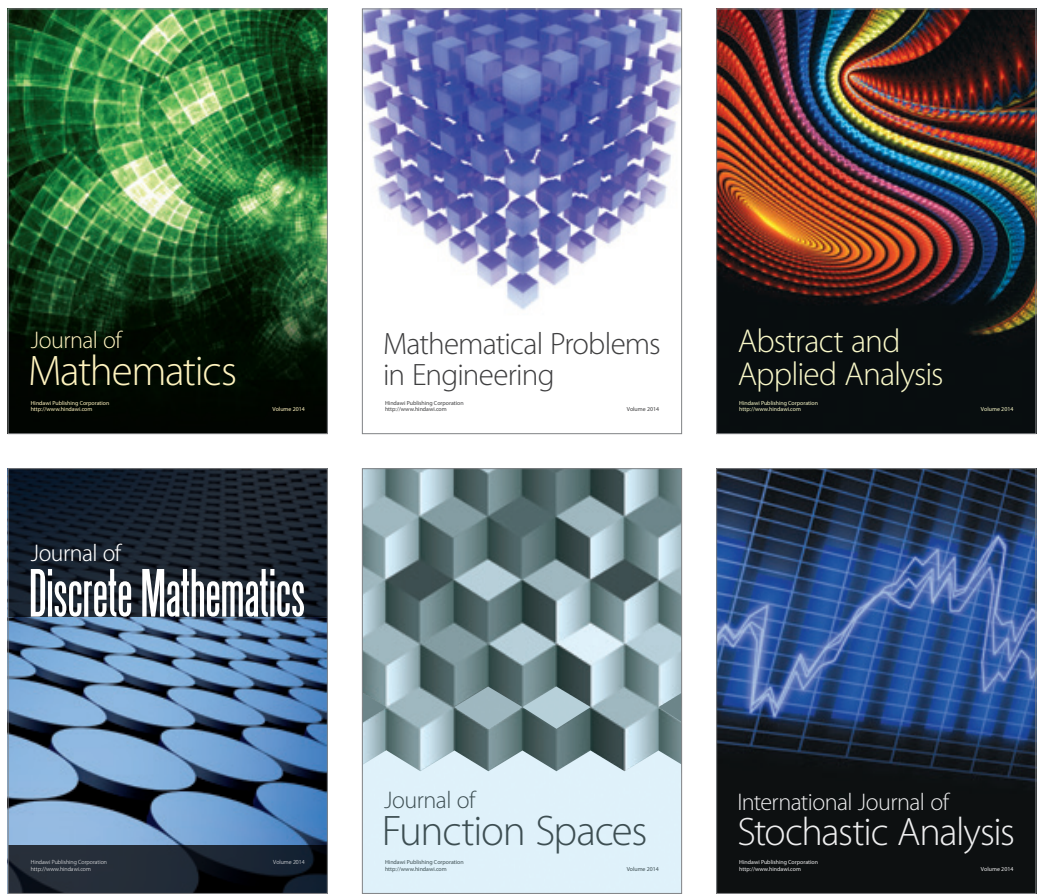

Journal of

Function Spaces

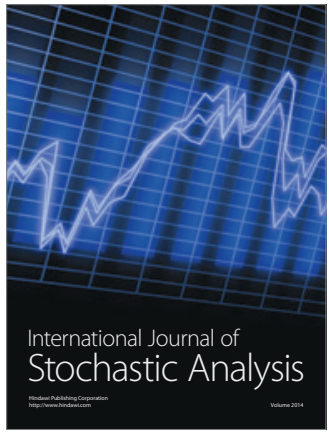

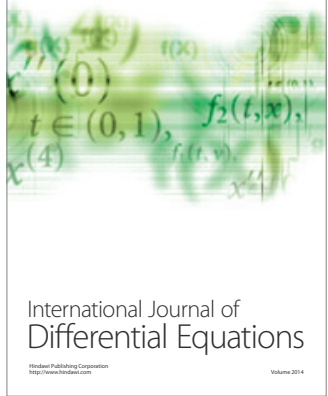
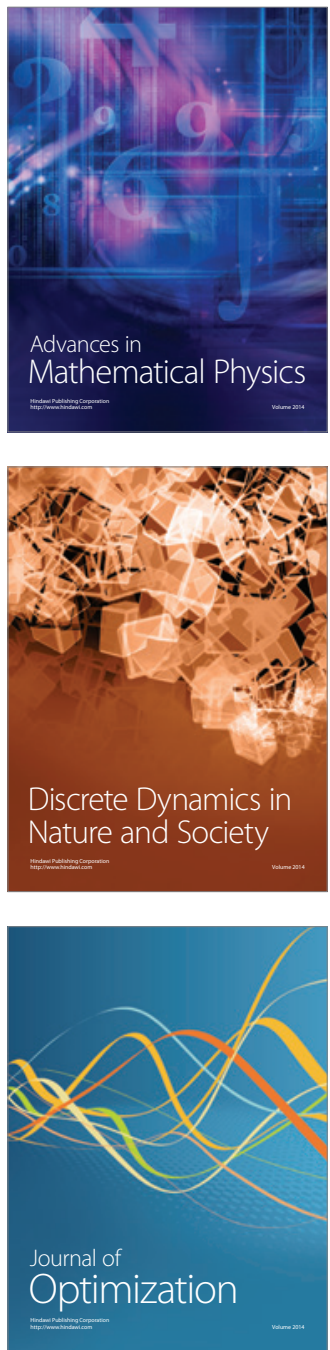